\title{
ON A PROOF OF A CONJECTURE OF MARIÑO-VAFA ON HODGE INTEGRALS
}

\author{
Chiu-Chu Melissa Liu, Kefeng Liu, and Jian Zhou
}

ABStract. We outline a proof of a remarkable formula for Hodge integrals conjectured by Mariño and Vafa [25] based on large $N$ duality.

\section{Introduction}

Let $\overline{\mathcal{M}}_{g, n}$ denote the Deligne-Mumford moduli stack of stable curves of genus $g$ with $n$ marked points. Let $\pi: \overline{\mathcal{M}}_{g, n+1} \rightarrow \overline{\mathcal{M}}_{g, n}$ be the universal curve, and let $\omega_{\pi}$ be the relative dualizing sheaf. The Hodge bundle

$$
\mathbb{E}=\pi_{*} \omega_{\pi}
$$

is a rank $g$ vector bundle over $\overline{\mathcal{M}}_{g, n}$ whose fiber of over $\left[C, x_{1}, \ldots, x_{n}\right] \in \overline{\mathcal{M}}_{g, n}$ is $H^{0}\left(C, \omega_{C}\right)$. Let $s_{i}: \overline{\mathcal{M}}_{g, n} \rightarrow \overline{\mathcal{M}}_{g, n+1}$ denote the section of $\pi$ which corresponds to the $i$-th marked point, and let

$$
\mathbb{L}_{i}=s_{i}^{*} \omega_{\pi}
$$

be the line bundle over $\overline{\mathcal{M}}_{g, n}$ whose fiber over $\left[C, x_{1}, \ldots, x_{n}\right] \in \overline{\mathcal{M}}_{g, n}$ is the cotangent line $T_{x_{i}}^{*} C$ at the $i$-th marked point $x_{i}$. A Hodge integral is an integral of the form

$$
\int_{\overline{\mathcal{M}}_{g, n}} \psi_{1}^{j_{1}} \cdots \psi_{n}^{j_{n}} \lambda_{1}^{k_{1}} \cdots \lambda_{g}^{k_{g}}
$$

where $\psi_{i}=c_{1}\left(\mathbb{L}_{i}\right)$ is the first Chern class of $\mathbb{L}_{i}$, and $\lambda_{j}=c_{j}(\mathbb{E})$ is the $j$-th Chern class of the Hodge bundle.

Hodge integrals arise in the calculations of Gromov-Witten invariants by localization techniques $[14,7]$. The explicit evaluation of Hodge integrals is a difficult problem. The Hodge integrals involving only $\psi$ classes can be computed recursively by Witten's conjecture [27] proven by Kontsevich [13]. Algorithms of computing Hodge integrals are described in [2].

In [25], M. Mariño and C. Vafa obtained a closed formula for a generating function of certain open Gromov-Witten invariants, some of which has been reduced to Hodge integrals by localization techniques which are not fully clarified mathematically. This leads to a conjectural formula of Hodge integrals. To state this formula, we introduce some notation. Let

$$
\Lambda_{g}^{\vee}(u)=u^{g}-\lambda_{1} u+\cdots+(-1)^{g} \lambda_{g}
$$

Received June 18, 2003. 
be the Chern polynomial of $\mathbb{E}^{\vee}$, the dual of the Hodge bundle. For a partition $\mu$ given by

$$
\mu_{1} \geq \mu_{2} \geq \cdots \geq \mu_{l(\mu)}>0,
$$

let $|\mu|=\sum_{i=1}^{l(\mu)} \mu_{i}$, and define

$$
\begin{aligned}
\mathcal{C}_{g, \mu}(\tau)= & -\frac{\sqrt{-1}|\mu|+l(\mu)}{|\operatorname{Aut}(\mu)|}[\tau(\tau+1)]^{l(\mu)-1} \prod_{i=1}^{l(\mu)} \frac{\prod_{a=1}^{\mu_{i}-1}\left(\mu_{i} \tau+a\right)}{\left(\mu_{i}-1\right) !} \\
& \cdot \int_{\overline{\mathcal{M}}_{g, l(\mu)}} \frac{\Lambda_{g}^{\vee}(1) \Lambda_{g}^{\vee}(-\tau-1) \Lambda_{g}^{\vee}(\tau)}{\prod_{i=1}^{l(\mu)}\left(1-\mu_{i} \psi_{i}\right)} \\
\mathcal{C}_{\mu}(\lambda ; \tau)= & \sum_{g \geq 0} \lambda^{2 g-2+l(\mu)} \mathcal{C}_{g, \mu}(\tau)
\end{aligned}
$$

Note that

$$
\int_{\overline{\mathcal{M}}_{0, l(\mu)}} \frac{\Lambda_{0}^{\vee}(1) \Lambda_{0}^{\vee}(-\tau-1) \Lambda_{0}^{\vee}(\tau)}{\prod_{i=1}^{l(\mu)}\left(1-\mu_{i} \psi_{i}\right)}=\int_{\overline{\mathcal{M}}_{0, l(\mu)}} \frac{1}{\prod_{i=1}^{l(\mu)}\left(1-\mu_{i} \psi_{i}\right)}=|\mu|^{l(\mu)-3}
$$

for $l(\mu) \geq 3$, and we use this expression to extend the definition to the case $l(\mu)<3$.

Introduce formal variables $p=\left(p_{1}, p_{2}, \ldots, p_{n}, \ldots\right)$, and define

$$
p_{\mu}=p_{\mu_{1}} \cdots p_{\mu_{l(\mu)}}
$$

for a partition $\mu=\left(\mu_{1} \geq \cdots \geq \mu_{l(\mu)}>0\right)$. Define generating functions

$$
\begin{aligned}
\mathcal{C}(\lambda ; \tau ; p) & =\sum_{|\mu| \geq 1} \mathcal{C}_{\mu}(\lambda ; \tau) p_{\mu}, \\
\mathcal{C}(\lambda ; \tau ; p)^{\bullet} & =e^{\mathcal{C}(\lambda ; \tau ; p)} .
\end{aligned}
$$

As pointed out in [25], by comparing computations in [25] with computations in [12], one obtains a conjectural formula for $\mathcal{C}_{\mu}(\tau)$. This formula is explicitly written down in [29]:

$\mathcal{C}(\lambda ; \tau ; p)=\sum_{n \geq 1} \frac{(-1)^{n-1}}{n} \sum_{\mu}\left(\sum_{\cup_{i=1}^{n} \mu^{i}=\mu} \prod_{i=1}^{n} \sum_{\left|\nu^{i}\right|=\left|\mu^{i}\right|} \frac{\chi_{\nu^{i}}\left(C\left(\mu^{i}\right)\right)}{z_{\mu^{i}}} e^{\sqrt{-1}\left(\tau+\frac{1}{2}\right) \kappa_{\nu^{i}} \lambda / 2} V_{\nu^{i}}(\lambda)\right) p_{\mu}$,

$$
\mathcal{C}(\lambda ; \tau ; p)^{\bullet}=\sum_{|\mu| \geq 0}\left(\sum_{|\nu|=|\mu|} \frac{\chi_{\nu}(C(\mu))}{z_{\mu}} e^{\sqrt{-1}\left(\tau+\frac{1}{2}\right) \kappa_{\nu} \lambda / 2} V_{\nu}(\lambda)\right) p_{\mu}
$$

where

$$
\begin{aligned}
V_{\nu}(\lambda)= & \prod_{1 \leq a<b \leq l(\nu)} \frac{\sin \left[\left(\nu_{a}-\nu_{b}+b-a\right) \lambda / 2\right]}{\sin [(b-a) \lambda / 2]} \\
& \cdot \frac{1}{\prod_{i=1}^{l(\nu)} \prod_{v=1}^{\nu_{i}} 2 \sin [(v-i+l(\nu)) \lambda / 2]}
\end{aligned}
$$


We now explain the notation on the right-hand sides of (1) and (2). For a partition $\mu$ given by

$$
\mu_{1} \geq \mu_{2} \geq \cdots \geq \mu_{l(\mu)}>0
$$

$\chi_{\mu}$ denotes the character of the irreducible representation of $S_{d}$ indexed by $\mu$, where $d=|\mu|=\sum_{i=1}^{l(\mu)} \mu_{i}$. The number $\kappa_{\mu}$ is defined by

$$
\kappa_{\mu}=|\mu|+\sum_{i}\left(\mu_{i}^{2}-2 i \mu_{i}\right)
$$

For each positive integer $i$,

$$
m_{i}(\mu)=\left|\left\{j: \mu_{j}=i\right\}\right| .
$$

Denote by $C(\nu)$ the conjugacy class of $S_{d}$ corresponding to the partition $\nu$, and by $\chi_{\mu}(C(\nu))$ the value of the character $\chi_{\mu}$ on the conjugacy class $C(\nu)$. Finally,

$$
z_{\mu}=\prod_{j} m_{j}(\mu) ! j^{m_{j}(\mu)}
$$

In this paper, we will call (1) the Mariño-Vafa formula.

The third author proved in [28] some special cases of the Mariño-Vafa formula and found some applications. In [30] it was shown that calculation of BPS numbers in the local $\mathbb{P}^{2}$ and $\mathbb{P}^{1} \times \mathbb{P}^{1}$ geometries can be reduced to the Mariño-Vafa formula. In [31] a special case of diagrammatic rule in [11] was proved assuming the Mariño-Vafa formula. The authors have shown in [23] that many known identities for Hodge integrals can be deduced from the Mariño-Vafa formula.

We now describe our approach to the Mariño-Vafa formula (1). Denote the right-hand sides of (1) and (2) by $R(\lambda ; \tau ; p)$ and $R(\lambda ; \tau ; p)^{\bullet}$ respectively. In [29], the third author proved the following two equivalent cut-and-join equations similar to the one satisfied by Hurwitz numbers [6], [16], [10, Section 15.2].

\section{Theorem 1.}

$$
\begin{aligned}
& \frac{\partial R}{\partial \tau}=\frac{\sqrt{-1} \lambda}{2} \sum_{i, j \geq 1}\left(i j p_{i+j} \frac{\partial^{2} R}{\partial p_{i} \partial p_{j}}+i j p_{i+j} \frac{\partial R}{\partial p_{i}} \frac{\partial R}{\partial p_{j}}+(i+j) p_{i} p_{j} \frac{\partial R}{\partial p_{i+j}}\right) \\
& \frac{\partial R^{\bullet}}{\partial \tau}=\frac{\sqrt{-1} \lambda}{2} \sum_{i, j \geq 1}\left(i j p_{i+j} \frac{\partial^{2} R^{\bullet}}{\partial p_{i} \partial p_{j}}+(i+j) p_{i} p_{j} \frac{\partial R}{\partial p_{i+j}}\right)
\end{aligned}
$$

Here is a crucial observation: One can rewrite (5) as a sequence of systems of ordinary equations, one for each positive integer $d$, hence if $\mathcal{C}(\lambda ; \tau ; p)^{\bullet}$ satisfies (5), then it is determined by the initial value $\mathcal{C}(\lambda ; 0 ; p)^{\bullet}$. To prove $(1)$ or $(2)$, it suffices to prove the following two statements:

(a) Equation (4) is satisfied by $\mathcal{C}(\lambda ; \tau ; p)$.

(b) $\mathcal{C}(\lambda ; 0 ; p)=R(\lambda ; 0 ; p)$.

Or equivalently,

(a)' Equation (5) is satisfied by $\mathcal{C}(\lambda ; \tau ; p)^{\bullet}$.

(b)' $\mathcal{C}(\lambda ; 0 ; p)^{\bullet}=R(\lambda ; 0 ; p)^{\bullet}$. 
It is shown in [29] that (b) holds. Therefore, the Mariño-Vafa formula (1) follows from the following theorem.

\section{Theorem 2.}

$$
\frac{\partial \mathcal{C}}{\partial \tau}=\frac{\sqrt{-1} \lambda}{2} \sum_{i, j \geq 1}\left(i j p_{i+j} \frac{\partial^{2} \mathcal{C}}{\partial p_{i} \partial p_{j}}+i j p_{i+j} \frac{\partial \mathcal{C}}{\partial p_{i}} \frac{\partial \mathcal{C}}{\partial p_{j}}+(i+j) p_{i} p_{j} \frac{\partial \mathcal{C}}{\partial p_{i+j}}\right)
$$

The rest of the paper is organized as follows. In Section 2, we give a proof of the initial condition (b). In Section 3, we give the proof of Theorem 1 in [29]. In Section 4, we outline the proof of Theorem 2 in [22]. The details we omit here are straightforward calculations which will be given in [22]. Complete lists of relevant references will be given in $[29,22]$.

\section{Initial Condition}

The proof of the initial condition (b) needs the following two theorems.

Theorem 2.1. We have

$$
\mathcal{C}(\lambda ; 0 ; p)=-\sum_{n>0} \frac{\sqrt{-1}^{n+1} p_{n}}{2 n \sin (n \lambda / 2)} .
$$

Proof. When $l(\mu)>1$, we clearly have

$$
\mathcal{C}_{\mu}(\lambda ; 0)=0 .
$$

When $\mu=(n)$ we have

$$
\begin{aligned}
\mathcal{C}_{(n)}(\lambda ; 0) & =-\sum_{g \geq 0} \lambda^{2 g-1} \sqrt{-1}^{n+1} \frac{\prod_{a=1}^{n-1}(n \cdot 0+a)}{(n-1) !} \int_{\overline{\mathcal{M}}_{g, 1}} \frac{\Lambda_{g}^{\vee}(1) \Lambda_{g}^{\vee}(0) \Lambda_{g}^{\vee}(-1)}{1-n \psi_{1}} \\
& =-\frac{\sqrt{-1}^{n+1}}{n} \sum_{g \geq 0}(n \lambda)^{2 g-1} \int_{\overline{\mathcal{M}}_{g, 1}} \lambda_{g} \psi_{1}^{2 g-2} \\
& =-\frac{\sqrt{-1}^{n+1}}{n^{2} \lambda} \cdot \frac{n \lambda / 2}{\sin (n \lambda / 2)} \\
& =-\frac{\sqrt{-1}^{n+1}}{2 n \sin (n \lambda / 2)} .
\end{aligned}
$$

In the second equality we have used the Mumford's relations [26, 5.4]:

$$
\Lambda_{g}^{\vee}(1) \Lambda_{g}^{\vee}(-1)=(-1)^{g} \text {. }
$$

In the third equality we have used [3, Theorem 2]. This proves (7).

Theorem 2.2. We have the following identity:

$$
\log \left(\sum_{n \geq 0} \sum_{|\rho|=n} \frac{e^{\frac{1}{4} \kappa_{\rho} \sqrt{-1} \lambda}}{\prod_{e \in \rho} 2 \sin (h(e) \lambda / 2)} \frac{\chi_{\rho}(\eta)}{z_{\eta}} p_{\eta}\right)=-\sum_{d \geq 1} \frac{\sqrt{-1}^{d+1} p_{d}}{2 d \sin (d \lambda / 2)} .
$$


To prove (8), we need the following two lemmas whose proofs can be found in $[22]$. For a partition $\eta$,

$$
n(\eta)=\sum_{i}(i-1) \eta_{i}=\sum_{i}\left(\begin{array}{c}
\eta_{i}^{\prime} \\
2
\end{array}\right)
$$

For any box $e \in \eta$, denote by $h(e)$ its hook length. Then

$$
\sum_{x \in \eta} h(x)=n(\eta)+n\left(\eta^{\prime}\right)+|\eta|
$$

Lemma 2.1. Introducing formal variables $x_{1}, \ldots, x_{n}, \ldots$ such that

$$
p_{i}\left(x_{1}, \ldots, x_{n}, \ldots\right)=x_{1}^{i}+\cdots+x_{n}^{i}+\cdots
$$

Then for for any positive integer $n$, we have

$$
\sum_{n \geq 0} t^{n} \sum_{|\rho|=n} \frac{q^{n(\rho)}}{\prod_{e \in \rho}\left(1-q^{h(e)}\right)} \frac{\chi_{\rho}(\eta)}{z_{\rho}} p_{\eta}=\frac{1}{\prod_{i, j}\left(1-t x_{i} q^{j-1}\right)}
$$

Lemma 2.2. For any partition $\rho$ we have

$$
\frac{1}{2} \sum_{e \in \rho} h(e)-n(\rho)=\frac{1}{4} \kappa_{\rho}+\frac{1}{2}|\rho| .
$$

Now let $q=e^{-\sqrt{-1} \lambda}$, and $t=\sqrt{-1} q^{1 / 2}$, then we have by (10):

$$
\begin{aligned}
& \sum_{n \geq 0} t^{n} \sum_{|\rho|=n} \frac{q^{n(\rho)}}{\prod_{e \in \rho}\left(1-q^{h(e)}\right)} \frac{\chi_{\rho}(\eta)}{z_{\rho}} p_{\eta} \\
= & \sum_{n \geq 0} \sqrt{-1}^{n} q^{n / 2} \sum_{|\rho|=n} \frac{q^{n(\rho)-\frac{1}{2} \Sigma_{e \in \rho} h(e)}}{\prod_{e \in \rho}\left(q^{-h(e) / 2}-q^{h(e) / 2}\right)} \frac{\chi_{\rho}(\eta)}{z_{\rho}} p_{\eta} \\
= & \sum_{n \geq 0} \sqrt{-1}^{n} q^{n / 2} \sum_{|\rho|=n} \frac{q^{-\frac{1}{4} \kappa_{\rho}-\frac{1}{2} n}}{\prod_{e \in \rho}\left(q^{-h(e) / 2}-q^{h(e) / 2}\right)} \frac{\chi_{\rho}(\eta)}{z_{\rho}} p_{\eta} \\
= & \sum_{n \geq 0} \sum_{|\rho|=n} \frac{e^{\frac{1}{2} f_{\rho}(2) \sqrt{-1} \lambda}}{\prod_{e \in \rho} 2 \sin (h(e) \lambda / 2)} \frac{\chi_{\rho}(\eta)}{z_{\rho}} p_{\eta} .
\end{aligned}
$$


Hence by (9),

$$
\begin{aligned}
& \log \left(\sum_{n \geq 0} \sum_{|\rho|=n} \frac{e^{\frac{1}{4} \kappa_{\rho} \sqrt{-1} \lambda}}{\prod_{e \in \rho} 2 \sin (h(e) \lambda / 2)} \frac{\chi_{\rho}(\eta)}{z_{\rho}} p_{\eta}\right) \\
= & \log \left(\sum_{n \geq 0} t^{n} \sum_{|\rho|=n} \frac{q^{n(\rho)}}{\prod_{e \in \rho}\left(1-q^{h(e)}\right)} \frac{\chi_{\rho}(\eta)}{z_{\rho}} p_{\eta}\right) \\
= & \log \frac{1}{\prod_{i, j}\left(1-t x_{i} q^{j-1}\right)}=\sum_{i, j \geq 1} \sum_{d \geq 1} \frac{1}{d} t^{d} q^{d(j-1)} x_{i}^{d} \\
= & \sum_{j \geq 1} \sum_{d \geq 1} \frac{1}{d} t^{d} q^{d(j-1)} p_{d}=\sum_{d \geq 1} \frac{p_{d}}{d} \frac{t^{d}}{1-q^{d}} \\
= & -\sum_{d \geq 0} \frac{\sqrt{-1} d+1 p_{d}}{2 d \sin (d \lambda / 2)} .
\end{aligned}
$$

By (8) we have

$$
R(\lambda ; 0 ; p)=\log \left(\sum_{n \geq 0} \sum_{|\rho|=n} \frac{\chi_{\rho}(\eta)}{z_{\eta}} e^{\frac{1}{4} \kappa_{\rho} \lambda} V_{\rho} p_{\eta}\right)=-\sum_{d \geq 1} \frac{\sqrt{-1}^{d+1} p_{d}}{2 d \sin (d \lambda / 2)},
$$

where we have used the following identity proved in [29]:

$$
V_{\mu}=\frac{1}{2^{l} \prod_{x \in \mu} \sin [h(x) \lambda / 2]}
$$

Hence (b) is proved.

\section{Proof of Theorem 1}

Recall

$$
c_{\mu}=\sum_{g \in C_{\mu}} g
$$

lies in the center of the group algebra $\mathbb{C} S_{d}$, hence it acts as a scalar $f_{\nu}(\mu)$ on any irreducible representation $R_{\nu}$. In other words, let $\rho: S_{d} \rightarrow$ End $R_{\nu}$ be the representation indexed by $\nu$, then

$$
\sum_{g \in C(\mu)} \rho_{\nu}(g)=f_{\nu}(\mu) \text { id }
$$

We need the following interpretation of $\kappa_{\nu}$ in terms of character:

$$
\kappa_{\nu}=2 f_{\nu}(C(2))
$$

See [24], p. 118, Example 7. Here we use $C(2)$ to denote the class of transpositions. The cut-and-join equations were first established for Hurwitz numbers (see e.g. [6]). Our approach is not much different. We need the following easy observation: 
Lemma 3.1. Suppose $h \in S_{d}$ has cycle type $\mu$. The product $C_{(2)} \cdot h$ is a sum of elements of $S_{d}$ whose type is either a cut or a join of $\mu$. More precisely, there are $\operatorname{ijm}_{i}(\mu) m_{j}(\mu)$ (when $i<j$ ) or $i^{2} m_{i}(\mu)\left(m_{i}(\mu)-1\right) / 2($ when $i=j)$ elements obtained from $h$ by joining an $i$-cycle in $h$ to a $j$-cycle in $h$, and there are $(i+j) m_{i+j}(\mu)($ when $i<j)$ or $i_{2 i}(\mu)$ (when $i=j$ ) elements obtained from $h$ by cutting an $(i+j)$-cycle into an $i$-cycle and a $j$-cycle.

For any $h \in S_{d}$ of cycle type $\mu$ we have

$$
\begin{aligned}
& \sum_{\mu} f_{\nu}(2) \frac{\chi_{\nu}(\mu)}{z_{\mu}} p_{\mu} \\
= & \sum_{\mu} \operatorname{tr}\left[f_{\nu}(2) \mathrm{id} \cdot \rho_{\nu}(h)\right] \cdot \prod_{i} \frac{p_{i}^{m_{i}(\mu)}}{i^{m_{i}(\mu)} m_{i}(\mu) !} \\
= & \sum_{\mu} \operatorname{tr}\left[\sum_{g \in C(2)} \rho_{\nu}(g) \cdot \rho_{\nu}(h)\right] \cdot \prod_{i} \frac{p_{i}^{m_{i}(\mu)}}{i^{m_{i}(\mu)} m_{i}(\mu) !} \\
= & \sum_{\mu} \operatorname{tr} \rho_{\nu}\left(\sum_{g \in C(2)} g \cdot h\right) \cdot \prod_{i} \frac{p_{i}^{m_{i}(\mu)}}{i^{m_{i}(\mu)} m_{i}(\mu) !} \\
= & \sum_{\mu}\left(\sum_{i<j}\left(\sum_{\eta \in J_{i, j}(\mu)} i j m_{i}(\mu) m_{j}(\mu) \chi_{\nu}(\eta)+\sum_{\eta \in C_{i, j}(\mu)}(i+j) m_{i+j}(\mu) \chi_{\nu}(\eta)\right)\right. \\
& \left.+\sum_{i}\left(\sum_{\eta \in J_{i, i}(\mu)} \frac{1}{2} i^{2} m_{i}(\mu)\left(m_{i}(\mu)-1\right) \chi_{\nu}(\eta)+\sum_{\eta \in C_{i, i}(\mu)} i m_{2 i}(\mu) \chi_{\nu}(\eta)\right)\right) \\
& \cdot \prod_{i} \frac{p_{i}^{m_{i}(\mu)}}{i^{m_{i}(\mu)} m_{i}(\mu) !} \\
= & \frac{1}{2} \sum_{i, j}\left((i+j) p_{i} p_{j} \frac{\partial}{\partial p_{i+j}}+i j p_{i+j} \frac{\partial}{\partial p_{i}} \frac{\partial}{\partial p_{j}}\right) \sum_{\eta} \frac{\chi_{\nu}(\eta)}{z_{\eta}} p_{\eta} .
\end{aligned}
$$

Here we have the following notations. Let $\mu, \eta$ be two partitions, both represented by Young diagrams. We write $\eta \in J_{i, j}(\mu)$ and $\mu \in C_{i, j}(\eta)$ if $\eta$ is obtained from $\mu$ by removing a row of length $i$ and a row of length $j$, then adding a row of length $i+j$. It follows that

$$
\begin{aligned}
& \frac{\partial R(\lambda ; \tau ; p)}{\partial \tau} \\
= & \frac{\sqrt{-1} \lambda}{2} \sum_{\mu, \nu}\left(f_{\nu}(2) \frac{\chi_{\nu}(C(\mu))}{z_{\mu}} p_{\mu}\right) e^{\sqrt{-1}\left(\tau+\frac{1}{2}\right) \kappa_{\nu} \lambda / 2} V_{\nu}(\lambda) \\
= & \frac{\sqrt{-1} \lambda}{2}\left(i j p_{i+j} \frac{\partial}{\partial p_{i}} \frac{\partial}{\partial p_{j}}+(i+j) p_{i} p_{j} \frac{\partial}{\partial p_{i+j}}\right) \sum_{\eta} \frac{\chi_{\nu}(\eta)}{z_{\eta}} p_{\eta} e^{\sqrt{-1}\left(\tau+\frac{1}{2}\right) \kappa_{\nu} \lambda / 2} V_{\nu}(\lambda) .
\end{aligned}
$$

This finishes the proof of Theorem 1 . 


\section{Proof of Theorem 2}

4.1. Moduli space of relative morphisms. We first describe the moduli space of stable relative morphisms to $\mathbb{P}^{1}$ used in [20]. The moduli spaces of algebraic stable relative morphisms are constructed by J. Li [17]. The construction in symplectic geometry was carried out by Li-Ruan [15] and Ionel-Parker [9, 10].

Let $\mathbb{P}^{1}[m]$ be a chain of $m+1$ copies $\mathbb{P}^{1}$, such that the $i$-th copy is glued to the $(i+1)$-th copy at the point $p_{1}^{(i)}$ for $i=0, \ldots, m-1$. The zeroth copy will be referred to as the root component, and the other components will be called the bubble components. A point $p_{1}^{(m)}$ is fixed on the $m$-th (and the last) copy of $\mathbb{P}^{1}$. Denote by $\pi[m]: \mathbb{P}^{1}[m] \rightarrow \mathbb{P}^{1}$ the map which is identity on the root component and contracts all the bubble components to $p_{1}^{(0)}$.

Let $\mu$ be a partition of $d>0$. Let $\overline{\mathcal{M}}_{g, 0}\left(\mathbb{P}^{1}, \mu\right)$ be the moduli space of morphisms

$$
f:\left(C, x_{1}, \ldots, x_{l(\mu)}\right) \rightarrow \mathbb{P}^{1}[m]
$$

such that

1. $\left(C, x_{1}, \ldots, x_{l(\mu)}\right)$ is a prestable curve of genus $g$ with $l(\mu)$ marked points. For later convenience, we assume the marked points are unordered.

2. $f^{-1}\left(p_{1}^{(m)}\right)=\sum_{i=1}^{l(\mu)} \mu_{i} x_{i}$ as Cartier divisors, and $\operatorname{deg}(\pi[m] \circ f)=d$.

3. The preimage of each node in $\mathbb{P}^{1}[m]$ consists of nodes of $C$. If $f(y)=p_{1}^{(i)}$ and $C_{1}$ and $C_{2}$ are two irreducible components of $C$ which intersects at $y$, then $\left.f\right|_{C_{1}}$ and $\left.f\right|_{C_{2}}$ has the same contact order to $p_{1}^{(i)}$ at $y$.

4. The automorphism group of $f$ is finite.

Two such morphisms are isomorphic if they differ by an isomorphism of the domain and an automorphism of $\left(\mathbb{P}^{1}(m), p_{1}^{(0)}, p_{1}^{(m)}\right)$, where $\mathbb{P}^{1}(m)$ is the union of bubble components. In particular, this defines the automorphism group in the stability condition (4) above.

In $[17,18], \mathrm{J} . \mathrm{Li}$ showed that $\overline{\mathcal{M}}_{g, 0}\left(\mathbb{P}^{1}, \mu\right)$ is a separated, proper DeligneMumford stack with a perfect obstruction theory of virtual dimension

$$
r=2 g-2+|\mu|+l(\mu),
$$

so it has a virtual fundamental class of degree $r$.

4.2. Torus action. Consider the $\mathbb{C}^{*}$-action

$$
t \cdot\left[z^{0}: z^{1}\right]=\left[t z^{0}: z^{1}\right]
$$

on $\mathbb{P}^{1}$. It has two fixed points $p_{0}=[0: 1]$ and $p_{1}=[1: 0]$. This induces an action on $\mathbb{P}^{1}[m]$ by the action on the root component induced by the isomorphism to $\mathbb{P}^{1}$, and the trivial actions on the bubble components. This in turn induces an action on $\overline{\mathcal{M}}_{g, 0}\left(\mathbb{P}^{1}, \mu\right)$. 
4.3. The branch morphism. There is a branch morphism

$$
\mathrm{Br}: \overline{\mathcal{M}}_{g, 0}\left(\mathbb{P}^{1}, \mu\right) \rightarrow \operatorname{Sym}^{r} \mathbb{P}^{1} \cong \mathbb{P}^{r}
$$

Note that $\mathbb{P}^{r}$ can be identified with $\mathbb{P}\left(H^{0}\left(\mathbb{P}^{1}, \mathcal{O}(r)\right)\right.$, and the isomorphism

$$
\mathbb{P}\left(H^{0}\left(\mathbb{P}^{1}, \mathcal{O}(r)\right) \cong \operatorname{Sym}^{r} \mathbb{P}^{1}\right.
$$

is given by $[s] \mapsto \operatorname{div}(s)$. The $\mathbb{C}^{*}$ action on $\mathbb{P}^{1}$ induces a $\mathbb{C}^{*}$ action on $H^{0}\left(\mathbb{P}^{1}, \mathcal{O}(r)\right)$ by

$$
t \cdot\left(z^{0}\right)^{k}\left(z^{1}\right)^{r-k}=t^{-k}\left(z^{0}\right)^{k}\left(z^{1}\right)^{r-k} .
$$

So $\mathbb{C}^{*}$ acts on $\mathbb{P}^{r}$ by

$$
t \cdot\left[a_{0}, a_{1}, \ldots, a_{r}\right]=\left[a_{0}, t^{-1} a_{1}, \ldots, t^{-r} a_{r}\right],
$$

where $\left(a_{0}, a_{1}, \ldots, a_{r}\right)$ corresponds to $\sum_{k=0}^{r} a_{k}\left(z^{0}\right)^{k}\left(z^{1}\right)^{r-k} \in H^{0}\left(\mathbb{P}^{1}, \mathcal{O}(r)\right)$. With this action, the branch morphism is $\mathbb{C}^{*}$-equivariant.

4.4. The obstruction bundle. In [20], J. Li and Y. Song constructed an obstruction bundle over the stratum where the target is $\mathbb{P}^{1}[0]=\mathbb{P}^{1}$, and proposed an extension over the entire $\overline{\mathcal{M}}_{g, 0}\left(\mathbb{P}^{1}, \mu\right)$. Here we use a different extension which is equivalent to the one used in [1].

Let $\pi[m]: \mathbb{P}^{1}[m] \rightarrow \mathbb{P}^{1}$ be the contraction to the root component, and denote $\tilde{f}=\pi[m] \circ f$. Dual to the obstruction space at a map $f:\left(C, x_{1}, \ldots, x_{l(\mu)}\right) \rightarrow$ $\mathbb{P}^{1}[m]$, consider the vector bundle $V$ with fiber at $f$ given by

$$
H^{1}\left(C, \mathcal{O}_{C}(-D)\right) \oplus H^{1}\left(C, \tilde{f}^{*} \mathcal{O}_{\mathbb{P}^{1}}(-1)\right),
$$

where $D=x_{1}+\ldots+x_{l(\mu)}$. It is a direct sum of two vector bundles $V_{D}$ and $V_{D_{d}}$.

Note that

$$
H^{0}\left(C, \mathcal{O}_{C}(-D)\right)=H^{0}\left(C, \tilde{f}^{*} \mathcal{O}_{\mathbb{P}^{1}}(-1)\right)=0
$$

so the ranks of $V_{D}$ and $V_{D_{d}}$ are, by Riemann-Roch, $l(\mu)+g-1$ and $d+g-1$, respectively.

We lift $\mathbb{C}^{*}$ action on $\overline{\mathcal{M}}_{g, 0}\left(\mathbb{P}^{1}, \mu\right)$ to $V_{D}$ and $V_{D_{d}}$ as follows. The action on $V_{D_{d}}$ comes from an action on $\mathcal{O}_{\mathbb{P}^{1}}(-1) \rightarrow \mathbb{P}^{1}$ with weights $-\tau-1$ and $-\tau$ at the two fixed points $p_{0}$ and $p_{1}$, respectively, where $\tau \in \mathbb{Z}$. The fiber of $V_{D}$ does not depend on the map $f$, so the fibers over two points in the same orbit of the $\mathbb{C}^{*}$ action can be canonically identified. The action of $\lambda \in \mathbb{C}^{*}$ on $V_{D}$ is multiplication by $\lambda^{\tau}$.

4.5. Functorial localization. Let $T=\mathbb{C}^{*}$. We will compute

$$
\mathrm{Br}_{*} e_{T}(V)=\sum_{l=0}^{r} a_{l}(\tau) H^{l} u^{r-l} .
$$

by virtual functorial localization $[21]$. Here $H \in H^{2}\left(\mathbb{P}^{r} ; \mathbb{Z}\right)$ is the hyperplane class, and $a_{l}(\tau)$ is a polynomial in $\tau$. 
Let $p_{k} \in \mathbb{P}^{r}$ be the $T$ fixed point which corresponds to $\mathbb{C}\left(z^{0}\right)^{k}\left(z^{1}\right)^{r-k} \subset$ $H^{0}\left(\mathbb{P}^{1}, \mathcal{O}(r)\right)$, and let $f_{k}: p_{k} \rightarrow \mathbb{P}^{r}$ be the inclusion. Let $F(\tau, x)=\sum_{l=0} a_{l}(\tau) x^{l}$. Then

$$
\frac{f_{k}^{*} \mathrm{Br}_{*} e_{T}(V)}{e_{T}\left(T_{p_{k}} \mathbb{P}^{r}\right)}=\frac{F(\tau, k)}{(-1)^{r-k} k !(r-k) !} .
$$

By functorial localization, we have

$$
\int_{p_{r-k}} \frac{f_{r-k}^{*} \mathrm{Br}_{*} e_{T}(V)}{e_{T}\left(T_{p_{r-k}} \mathbb{P}^{r}\right)}=\sum_{F \subset \mathrm{Br}^{-1}\left(p_{r-k}\right)} \int_{[F]^{v i r}} \frac{i_{F}^{*} e_{T}(V)}{e_{T}\left(N_{F}^{v i r}\right)}
$$

for $k=0, \ldots, r$, where $N_{F}^{v i r}$ is the virtual normal bundle of the fixed loci $F$ in $\overline{\mathcal{M}}_{g, 0}\left(\mathbb{P}^{1}, \mu\right)$, and

$$
i_{F}: F \rightarrow \overline{\mathcal{M}}_{g, 0}\left(\mathbb{P}^{1}, \mu\right)
$$

is the inclusion. It is computed in [22] that

$$
\sum_{F \subset \operatorname{Br}^{-1}\left(p_{r-k}\right)} \int_{[F] \text { vir }} \frac{i_{F}^{*} e_{T}(V)}{e_{T}\left(N_{F}^{v i r}\right)}=\tau^{k} J_{g, \mu}^{k}(\tau),
$$

where $J_{g, \mu}^{k}(\tau)$ is a degree $r-k$ polynomial in $\tau$, and

$$
J_{g, \mu}^{k}(-\tau-1)=(-1)^{d-l(\mu)+k} J_{g, \mu}^{k}(\tau) .
$$

Moreover, we have

$$
\begin{aligned}
J_{g, \mu}^{0}(\tau)= & \sqrt{-1}^{|\mu|-l(\mu)} \mathcal{C}_{g, \mu}(\tau), \\
J_{g, \mu}^{1}(\tau)= & \sqrt{-1}^{|\mu|-l(\mu)-1}\left(\sum_{\nu \in J(\mu)} I_{1}(\nu) \mathcal{C}_{g, \nu}(\tau)+\sum_{\nu \in C(\mu)} I_{2}(\nu) \mathcal{C}_{g, \nu}(\tau)\right. \\
& \left.+\sum_{g_{1}+g_{2}=g} \sum_{\nu^{1} \cup \nu^{2} \in C(\mu)} I_{3}\left(\nu^{1}, \nu^{2}\right) \mathcal{C}_{g_{1}, \nu^{1}}(\tau) \mathcal{C}_{g_{2}, \nu^{2}}(\tau)\right) .
\end{aligned}
$$

Here we use the notation in [16]. The set $J(\mu)$ (join) consists of partitions of $d$ of the form

$$
\nu=\left(\mu_{1}, \ldots, \hat{\mu}_{i}, \ldots, \hat{\mu}_{j}, \ldots, \mu_{l(\mu)}, \mu_{i}+\mu_{j}\right)
$$

and the set $C(\mu)$ (cut) consists of partitions of $d$ of the form

$$
\nu=\left(\mu_{1}, \ldots, \hat{\mu}_{i}, \ldots, \mu_{l(\mu)}, j, k\right)
$$

where $j+k=\mu_{i}$. The precise definitions of $I_{1}, I_{2}$, and $I_{3}$ can be found in [16]. It follows from the definition that (6) in Theorem 2 is equivalent to

$$
\frac{d}{d \tau} J_{g, \mu}^{0}(\tau)=-J_{g, \mu}^{1}(\tau) .
$$


Since

$$
\begin{aligned}
& F(\tau, x) \\
& =\sum_{k=0}^{r} \frac{F(\tau, k)}{(-1)^{r-k} k !(r-k) !} x(x-1) \cdots(x-k+1)(x-k-1) \cdots(x-r) \\
& =\sum_{k=0}^{r} \tau^{r-k} J_{g, \mu}^{r-k}(\tau) x(x-1) \cdots(x-k+1)(x-k-1) \cdots(x-r) \\
& =\sum_{k=0}^{r} \tau^{k} J_{g, \mu}^{k}(\tau) x(x-1) \cdots(x-(r-k-1))(x-(r-k+1)) \cdots(x-r),
\end{aligned}
$$

therefore,

$\mathrm{Br}_{*} e_{T}(V)=\sum_{k=0}^{r} \tau^{k} J_{g, \mu}^{k}(\tau) H(H-u) \cdots(H-(r-k-1) u)(H-(r-k+1) u) \cdots(H-r u)$.

4.6. Final Calculations. For $i=0, \ldots, r-1$, we have

$$
\begin{aligned}
& H^{i} H(H-u) \cdots(H-(r-k-1) u)(H-(r-k+1) u) \cdots(H-r u) \\
= & ((H-(r-k) u)+(r-k) u)^{i} H(H-u) \cdots(H-(r-k-1) u) \\
& \cdot(H-(r-k+1) u) \cdots(H-r u) \\
= & ((r-k) u)^{i} H(H-u) \cdots(H-(r-k-1) u)(H-(r-k+1) u) \cdots(H-r u)
\end{aligned}
$$

since

$$
H(H-u) \cdots(H-r u)=0
$$

Therefore,

$$
\int_{\mathbb{P}^{r}} \mathrm{Br}_{*} e_{T}(V) H^{i}=u^{i} \sum_{k=0}^{r}(r-k)^{i} \tau^{k} J_{g, \mu}^{k}(\tau) .
$$

Let $J_{g, \mu}^{k}(\tau)=\sum_{j=0}^{r-k} a_{j}^{k} \tau^{j}$. We have

$$
u^{-i} \int_{\mathbb{P}^{r}} \operatorname{Br}_{*} e_{T}(V) H^{i}=\sum_{l=0}^{r}\left(\sum_{j+k=l}(r-k)^{i} a_{j}^{k}\right) \tau^{l} .
$$

Here is a crucial observation: as a polynomial in $\tau, u^{-i} \int_{\mathbb{P} r} \mathrm{Br}_{*} e_{T}(V) H^{i}$ is of degree no more than $i$. Therefore,

$$
\sum_{j+k=l}(r-k)^{i} a_{j}^{k}=0
$$

for $0 \leq i<l \leq r$. Now fix $l$ such that $1 \leq l \leq r$. We have

$$
\sum_{k=0}^{l}(r-k)^{i} a_{l-k}^{k}=0, \quad 0 \leq i<l,
$$

which is a system of $l$ linear equations of the $l+1$ variables $\left\{a_{l-k}^{k}: k=0, \ldots, l\right\}$. 
Both

$$
\left\{(r-t)^{i}: i=0, \ldots, l-1\right\}
$$

and

$$
\{1, t, t(t-1), \ldots, t(t-1) \ldots(t-l+2)\}
$$

are bases of the vector space

$$
\{f(t) \in \mathbb{Q}[t]: \operatorname{deg}(f) \leq l-1\}
$$

so there exists an invertible $l \times l$ matrix $\left(A_{i j}\right)_{0 \leq i, j \leq l-1}$ such that

$$
t(t-1) \cdots(t-i+1)=\sum_{j=0}^{l-1} A_{i j}(r-t)^{j} .
$$

In particular,

$$
k(k-1) \cdots(k-i+1)=\sum_{j=0}^{l-1} A_{i j}(r-k)^{j} .
$$

for $k=0,1, \ldots, l$, so (11) is equivalent to

$$
\sum_{k=0}^{l} k(k-1) \cdots(k-i+1) a_{l-k}^{k}=0, \quad 0 \leq i<l,
$$

i.e.,

$$
\sum_{k=i}^{l} \frac{k !}{(k-i) !} a_{l-k}^{k}=0, \quad 0 \leq i<l .
$$

The above equations can be rewritten as

$$
\left(\begin{array}{ccccccc}
1 & 1 & \ldots & \ldots & \ldots & \ldots & 1 \\
0 & 1 ! & 2 & \ldots & \ldots & \ldots & l \\
0 & 0 & 2 ! & 3 \cdot 2 & \ldots & \ldots & l(l-1) \\
0 & 0 & 0 & 3 ! & \ldots & \ldots & l(l-1)(l-2) \\
0 & \vdots & \vdots & \vdots & \ddots & \vdots & \vdots \\
0 & \cdots & \ldots & \ldots & 0 & (l-1) ! & l(l-1) \cdots 2
\end{array}\right)\left(\begin{array}{c}
a_{l}^{0} \\
a_{l-1}^{1} \\
\vdots \\
\vdots \\
a_{0}^{l}
\end{array}\right)=\left(\begin{array}{c}
0 \\
\vdots \\
\vdots \\
\vdots \\
0
\end{array}\right)
$$

The kernel is clearly one dimensional. One can check that the kernel is given by

$$
a_{l-k}^{k}=(-1)^{k} \frac{l !}{k !(l-k) !} a_{l}^{0} .
$$

Note that $(12)$ for $l=1, \ldots, r$ is equivalent to

$$
J_{g, \mu}^{k}(\tau)=\frac{(-1)^{k}}{k !} \frac{d^{k}}{d \tau^{k}} J_{g, \mu}^{0}(\tau)
$$

for $k=0, \ldots, r$. In particular,

$$
J_{g, \mu}^{1}(\tau)=-\frac{d}{d \tau} J_{g, \mu}^{0}(\tau)
$$

which is equivalent to the cut-and-join equation (6) in Theorem 2. 


\section{Acknowledgements}

We wish to thank Jun Li for explaining his work, Jim Bryan, Bohui Chen, Tom Graber, Gang Liu for helpful conversations, and Cumrun Vafa and ShingTung Yau for their interests in this work. The first author wishes to thank the hospitality of IPAM where she was a core participant of the Symplectic Geometry and Physics Program and did most of her part of this work. The second author is supported by an NSF grant. The third author is partially supported by research grants from NSFC and Tsinghua University.

\section{References}

[1] J. Bryan and R. Pandharipande, in preparation.

[2] C. Faber, Algorithms for computing intersection numbers on moduli spaces of curves, with an application to the class of the locus of Jacobians. London Math. Soc. Lecture Note Ser., 264, Cambridge Univ. Press, Cambridge, 1999.

[3] C. Faber, R. Pandharipande, Hodge integrals and Gromov-Witten theory. Invent. Math. 139 (2000), 173-199.

[4] _ Relative maps and tautological classes, Preprint, math.AG/0304485.

[5] B. Fantechi, R. Pandharipande, Stable maps and branch divisors, Compositio Math. 130 (2002), 345-364.

[6] I.P. Goulden, D.M. Jackson, A. Vainshtein, The number of ramified coverings of the sphere by the torus and surfaces of higher genera, Ann. of Comb. 4 (2000), 27-46.

[7] T. Graber, R. Pandharipande, Localization of virtual classes. Invent. Math. 135 (1999), $487-518$.

[8] T. Graber, R. Vakil, Hodge integrals and Hurwitz numbers via virtual localization. Compositio Math. 135 (2003), 25-36.

[9] E.-N. Ionel, T. Parker, Relative Gromov-Witten invaraints, Ann. of Math. 157 (2003), $45-96$.

[10] , The Symplectic Sum Formula for Gromov-Witten Invariants, Preprint, math.SG/0010217.

[11] A. Iqbal, All genus topological amplitudes and 5-brane webs as Feynman diagrams, Preprint, hep-th/0207114.

[12] S. Katz, C.-C. Liu, Enumerative geometry of stable maps with Lagrangian boundary condtions and multiple covers of the disc, Adv. Theor. Math. Phys. 5 (2001), 1-49.

[13] M. Kontsevich, Intersection theory on the moduli space of curves and the matrix Airy function. Comm. Math. Phys. 147 (1992), 1-23.

[14] M. Kontsevich, Enumeration of rational curves via torus actions. Progr. Math., 129, Birkhäuser Boston, Boston, MA, 1995.

[15] A.M. Li, Y. Ruan, Symplectic surgery and Gromov-Witten invariants of calabi-Yau 3folds, Invent. Math. 145 (2001), 151-218.

[16] A.M. Li, G. Zhao, Q. Zheng, The number of ramified coverings of a Riemann surface by Riemann surface, Comm. Math. Phys. 213 (2000), 685-696.

[17] J. Li, Stable Morphisms to singular schemes and relative stable morphisms, J. Diff. Geom. 57 (2001), 509-578.

[18] _ Relative Gromov-Witten invariants and a degeneration formula of GromovWitten invariants, J. Diff. Geom. 60 (2002), 199-293.

[19] Lecture notes on relative $G W$-invariants, preprint.

[20] J. Li, Y.S. Song, Open string instantons and relative stable morphisms. Adv. Theor. Math. Phys. 5 (2001), 67-91.

[21] B. Lian, K. Liu, S.T. Yau, Mirror Principle III, Asian J. Math. 3 (1999), 771-800. 
[22] C.-C. Liu, K. Liu, J. Zhou, A proof of a conjecture of Mariño-Vafa, to appear in J. Diff. Geom., math.AG/0306434.

[23] _ Mariño-Vafa and Hodge integrals, preprint, math.AG/0308015.

[24] I.G. MacDonald, Symmetric functions and Hall polynomials, 2nd edition.Claredon Press, 1995.

[25] M. Mariño, C. Vafa, Framed knots at large N, Contemp. Math., 310, Amer. Math. Soc., Providence, RI, 2002.

[26] D. Mumford, Towards an eunumerative geometry of the moduli space of curves, Birkhäuser, 1983, 271-328.

[27] E. Witten, Two-dimensional gravity and intersection theory on moduli space. Lehigh Univ., Bethlehem, PA, 1991.

[28] J. Zhou, Some closed formulas and conjectures for Hodge integrals, Math. Res. Lett. 10 (2003), 275-286.

[29] Hodge integrals, Hurwitz numbers, and symmetric groups, preprint, math.AG/0308024.

[30] _ Mariño-Vafa formula and BPS numbers in local $\mathbb{P}^{2}$ and $\mathbb{P}^{1} \times \mathbb{P}^{1}$ geometry, preprint, submitted.

[31] _ Proof and intepretation of a string duality, to appear in Math. Res. Lett.

Department of Mathematics, Harvard University, Cambridge, MA 02138, USA

E-mail address: ccliu@math.harvard.edu

Department of Mathematics, UCLA, Los Angeles, CA 90095-1555

E-mail address: liu@math.ucla.edu

Department of Mathematical Sciences, Tsinghua University, Beijing, 100084, China

E-mail address: jzhou@math.tsinghua.edu.cn 